\title{
TPX2 regulates tumor growth in human cervical carcinoma cells
}

\author{
PEIYUE JIANG ${ }^{1}$, KEXIN SHEN $^{2}$, XUERUI WANG $^{3}$, HAIQIN SONG $^{2}$, YING YUE $^{1}$ and TONGJUN LIU ${ }^{2}$ \\ ${ }^{1}$ Department of Gynecological Oncology, First Hospital of Jilin University, Changchun, Jilin 130021; \\ ${ }^{2}$ Department of Colorectal and Anal Surgery; ${ }^{3}$ Department of Ophthalmology, \\ China-Japan Union Hospital of Jilin University, Changchun, Jilin 130033, P.R. China
}

Received August 6, 2013; Accepted February 20, 2014

DOI: $10.3892 / \mathrm{mmr} .2014 .2106$

\begin{abstract}
The targeting protein for the Xenopus kinesin-like protein 2 (TPX2), a microtubule-associated protein, has been utilized as a tool to evaluate, more precisely, the proliferative behavior of tumor cells. The abnormal expression of TPX2 in a variety of malignant tumor types has been reported, however less is known about its role in cervical cancer. In the present study, the association between TPX2 expression and the biological behavior of cervical cancer, was investigated. Immunohistochemistry and RT-PCR were used to detect the expression of TPX2 in cervical cancer tissues. The inhibitory effect of TPX2-siRNA on the growth of SiHa human cervical carcinoma cells was studied in vitro. TPX2 expression was identified as significantly higher in cervical carcinoma compared with the control, normal cervical tissues. TPX2 siRNA transfected into SiHa cells induced apoptosis and inhibited cell proliferation and invasion. Similar results were obtained by in vivo transplantation, as TPX2 siRNA transfection significantly reduced tumor growth of the xenograft in nude mice. The results demonstrated that TPX2 is important in the regulation of tumor growth in cervical cancer and therefore may be a potential therapeutic target as a novel treatment strategy.
\end{abstract}

\section{Introduction}

Targeting protein for Xenopus kinesin-like protein 2 (TPX2) is a microtubule-associated protein. It is one of the best-known factors regulated by the RanGTP gradient and has a functional role in mitosis. The appearance and subsequent expression of

Correspondence to: Professor Ying Yue, Department of Gynecological Oncology, First Hospital of Jilin University, Changchun, 71 Xinmin Street, Jilin 130021, P.R. China

Email: yingyueuic@hotmail.com

Dr Tongjun Liu, Department of Colorectal and Anal Surgery, China-Japan Union Hospital of Jilin University, 126 Xiantai Street, Changchun, Jilin 130033, P.R. China

E-mail: tongjun.liu.2013@gmail.com

Key words: TPX2, cervical cancer, SiHa, siRNA, xenograft
TPX2 is mediated by the cell cycle, emerging at G1-S stage and diminishing following the completion of cytokinesis (1).

In recent studies, it has been revealed that TPX2 is overexpressed in various carcinoma tissue types, including lung (2), breast (3) and salivary gland cancer (4). In this study, it was demonstrated that the overexpression of TPX 2 in tumor cells caused exuberant amplification of the centrosome, developed aneuploidy and transformation, promotion of tumor proliferation and differentiation, as well as downregulation of tumor apoptosis (2-6). Conversely, it has also been demonstrated that inhibiting TPX2 and its associated pathways in tumor cells, leads to cancer cell apoptosis. This provides evidence that TPX2 may be a potential therapeutic candidate for the development of novel pharmacological cancer treatments $(5,7,8)$.

One recent study suggested that TPX 2 may also be expressed in cervical carcinoma (8), however the exact function of TPX2 in cervical cancer formation and regulation remains elusive. In the present study, the expression of TPX2 in cervical carcinoma in human patients and the human cervical cancer cell line $\mathrm{SiHa}$ cells was examined. Gene-silencing methods were utilized to specifically knock-down TPX2 expression in vitro and in vivo, to advance the understanding of the regulatory mechanisms of TPX2 in cervical cancer development. Our results may provide invaluable experimental data, to facilitate in the diagnosis and treatment of cervical cancer in the future.

\section{Materials and methods}

Clinical sample preparation. The specimens of cervical cancer tissues were collected from 52 patients at The China-Japan Union Hospital of Jilin University from May 2011-October 2012, which were immediately cryopreserved in liquid nitrogen and stored at $-80^{\circ} \mathrm{C}$. The cervical cancer patients' age was between 36-64 years (mean, 50.4 \pm 4.3 ) and the paraffin blocks of cervical samples were obtained. The normal tissue group had 24 specimens collected from the tissues of total hysterectomy due to myoma of the uterus and the patients' age range was 30-61 years (mean, 47.8 \pm 5.5 ). All patients provided informed consent and the experimental procedures were reviewed and approved by the Ethics Committee of the China-Japan Union Hospital of Jilin University (Changchun, Jilin, China).

$R T-P C R$. Total RNA was isolated using a TRIzol reagent kit (Invitrogen Life Technologies, Carlsbad, CA, USA) according to manufacturer's instructions. Reverse transcription was 

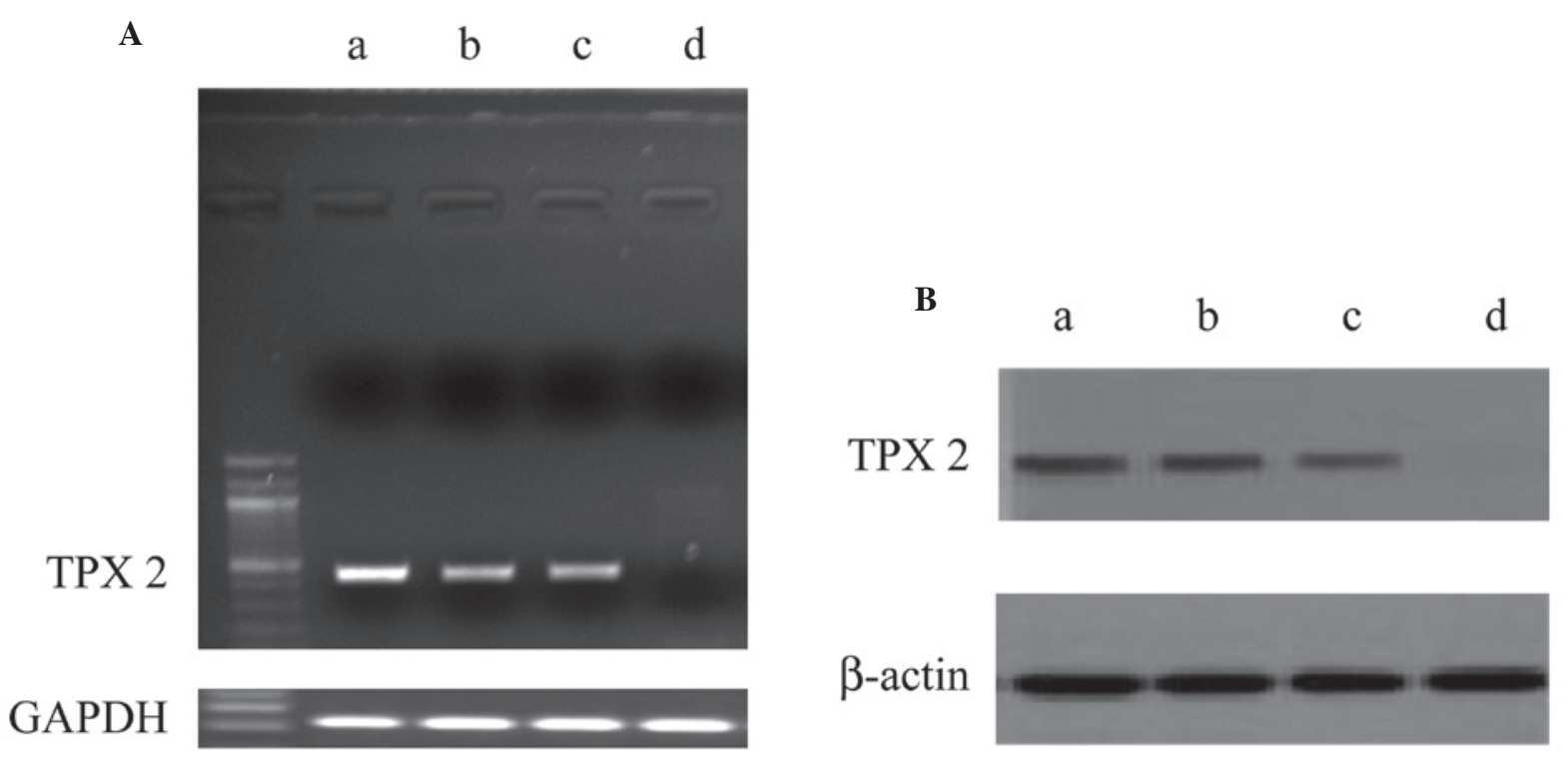

Figure 1. Expression of TPX2 in cervical tissues. (A) PCR. (B) Western blot analysis. In (A) and (B); lane a, cervical squamous cell carcinoma; lane b, SiHa cells; lane c, HeLa cells; lane d, normal cervical epithelium tissues. TPX2, targeting protein for Xenopus kinesin-like protein 2.

performed using a TaqMan microRNA reverse transcription kit (Applied Biosystems, Grand Island, NY, USA) according to the manufacturer's instructions. The coding sequences for TPX2 primers were: F, 5'-AACAATCCATTCCGTCAA-3' and R, 5'-TGCAGGTGGCATACAAGG-3'; GAPDH primers were: 5'-ACCTGACCTGCCGTCTAGAA-3' and 5'-TCCACCACCCTGTTGCTGTA-3'. cDNA amplification was performed in $25 \mu 1$ reaction tubes containing $0.2 \mu \mathrm{M}$ dNTPs, 20 pmol of each primer and $0.2 \mathrm{U}$ Tag polymerase in the PCR buffer.

Western blotting. Whole-cell collection was conducted by RIPA buffer [50 mM Tris, $150 \mathrm{mM} \mathrm{NaCl,} 1 \%$ Triton X-100, $0.1 \%$ sodium dodecyl sulfate and $1 \%$ Nadeoxycholate ( $\mathrm{pH} 7.4)$ ] supplemented with a protease inhibitor. Protein concentrations were then measured using Bio-Rad protein assay kits (Bio-Rad, Hercules, CA, USA). Then, the protein lysates were resolved by sodium dodecyl sulfate-polyacrylamide gel electrophoresis and transferred onto nitrocellulose membranes (Hybond ${ }^{\mathrm{TM}}-\mathrm{P}$; Amersham Biosciences, Piscataway, NJ, USA), blocked by PBS containing $0.2 \%$ Tween-20 and 5\% non fat dry milk, incubated with primary antibody and then with horseradish peroxidaselabeled secondary antibody. The signals were then detected by $\mathrm{X}$-ray film.

Apoptosis assay. Following 24-72 $\mathrm{h}$ in culture, $1 \times 10^{6}$ of the gastric cancer cells were washed twice with PBS and then resuspended in binding buffer $(10 \mathrm{mM}$ HEPES/NaOH, $140 \mathrm{mM}$ $\mathrm{NaCl}$, $2.5 \mathrm{mM} \mathrm{CaCl}_{2}$ ). FITC-Annexin $\mathrm{V}$ (Becton-Dickinson, Franklin Lakes, NJ, USA) was added at a final concentration of $1 \mathrm{mg} / \mathrm{ml}$ Annexin $\mathrm{V}$ and then $10 \mathrm{mg} / \mathrm{ml}$ PI was added. The mixture was incubated for $10 \mathrm{~min}$ in the dark at room temperature and then measured by a FACScan using Cellquest software (Becton-Dickinson).

Cell proliferation assay. Cells were plated at a concentration of $2.5 \times 10^{4}$ cells $/ \mathrm{ml}$ of culture medium in 96 -well plates. After
24,48 and $72 \mathrm{~h}$, the number of viable cells was determined in triplicate wells using an MTT assay (Sigma, St. Louis, MO, USA) according to the manufacturer's instructions.

Matrigel invasion assay. Migration assay was performed using a quantitative cell migration assay kit (ECM500; Chemicon, Temecula, CA, USA) according to the manufacturer's instructions. Warm serum-free medium $(200 \mathrm{ml})$ was added to the ECM layer and allowed to hydrate for 1-2 $\mathrm{h}$ at room temperature. Cells were dislodged following a brief trypsinization and dispersed into a homogeneous single-cell suspension that was washed and resuspended in serum-free medium at $5 \times 10^{5}$ cells $/ \mathrm{ml}$. Aliquots $(200 \mathrm{ml})$ of cell suspension were allowed to adhere to the surface for $1 \mathrm{~h}$ at $37^{\circ} \mathrm{C}$. The migration medium $(500 \mu \mathrm{l})$ containing cyclopamine was then added to the bottom chamber. Following $24 \mathrm{~h}$ incubation at $37^{\circ} \mathrm{C}$, with $5 \% \mathrm{CO}_{2}$ in the air, cells in the upper chamber were stained for $20 \mathrm{~min}$ and dissolved in $10 \%$ acetic acid and the optical density (OD) was read at $560 \mathrm{~nm}$ on a standard microplate reader.

siRNA transfection. Non-transfected cells were used as a control. Human TPX2 siRNA and scrambled siRNA (negative control) were purchased from IDT Inc. (Coralville, IA, USA). $\mathrm{SiHa}$ cells were plated in 6-well culture plates at a density of $2 \times 10^{5}$ cells/well. Following incubation overnight, cells were transfected with TPX2-siRNA $(50 \mathrm{nM})$ or the scrambled siRNA using GeneSilencer (Genlentis, CA, USA) according to the manufacturer's instructions.

Cervical xenograft. The SiHa cell suspension, including non-transfected and transfected with scrambled siRNA or TPX2-siRNA, (50 $\mu 1$ of $5 \times 10^{5}$ cells) were injected into the gastrocnemius muscle of 4 female SCID mice $24 \mathrm{~h}$ after siRNA transfection. Thirty days after grafting, nitroimidazole hypoxic marker EF5 (Ben Venue Laboratories, Bedford, $\mathrm{OH}, \mathrm{USA})$ was injected via a lateral tail vein $(200 \mu \mathrm{l}$ of a 

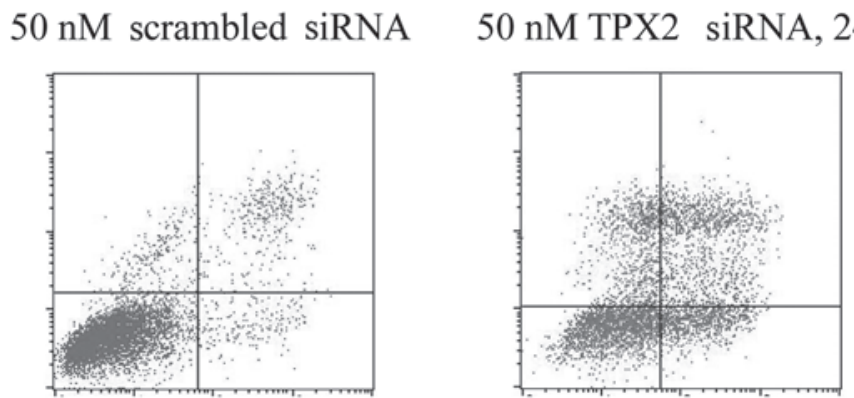

50 nM TPX2 siRNA, $72 \mathrm{~h}$

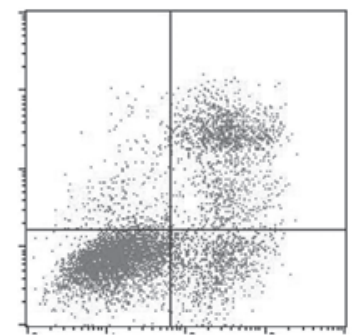

Figure 2. Apoptosis in SiHa cells by TPX2 siRNA. Evaluation of apoptosis by annexin V-FITC/PI staining and analysis by flow cytometry in SiHa cells treated with $50 \mathrm{nM}$ scrambled siRNA or $50 \mathrm{nM}$ TPX2 siRNA ( 24 and $72 \mathrm{~h}$ ). The results demonstrated TPX2 siRNA induced significant apoptosis in SiHa cells. TPX2, targeting protein for Xenopus kinesin-like protein 2.

$10 \mathrm{mM}$ stock solution) to give a total body concentration of $100 \mu \mathrm{M}$, followed by tumor extraction and immediate examination under light microscope (Nikon Eclipse E600; Nikon, Tokyo, Japan).

\section{Results}

TPX2 expression in cervical cancer tissues in vivo and in vitro. The mRNA and protein expression of TPX2 in pathological and normal cervical tissues were examined by RT-PCR and western blotting (Fig. 1). The results revealed that TPX2 mRNA and protein expression increased in cervical squamous cell carcinoma in vivo and cervical cancer cell line $\mathrm{SiHa}$ and HeLa cells in vitro. By contrast, there were weak/undetectable levels of TPX2 expression in normal cervical epithelium tissues in vivo.

TPX2 knockdown induces apoptosis in SiHa cells. SiHa cells were either transfected with $50 \mathrm{nM}$ scrambled siRNA or $50 \mathrm{nM}$ TPX2-siRNA for 24-72 h. The cells were then harvested and the apoptosis rates were analyzed using flow cytometry (Fig. 2). The results demonstrated that the cells treated with TPX2 silencing exhibited significantly higher apoptosis rates, at 24 and $72 \mathrm{~h}$, compared with the rate of the cells treated with the negative control siRNA.

TPX2 silencing inhibits cervical cancer proliferation. SiHa cells were either untransfected (control), transfected with $50 \mathrm{nM}$ scrambled siRNA or transfected with $50 \mathrm{nM}$ TPX2 siRNA. Cells in the three groups were then harvested at 24, 48 , and $72 \mathrm{~h}$ following transfection. The proliferation rate of SiHa cells was significantly lower in the TPX2-siRNA group compared with the control group and negative siRNA group $\left({ }^{*} \mathrm{P}<0.01\right.$; Fig. 3$)$. There was no statistically significant difference between the control group and negative siRNA group $(\mathrm{P}>0.05)$.

TPX2 silencing slows cervical cancer cell migration. SiHa cells were either untransfected (control), transfected with $50 \mathrm{nM}$ scrambled siRNA or transfected with $50 \mathrm{nM}$ TPX2 siRNA. The invasive capacity of SiHa cells was examined by a Matrigel invasion assay (Fig. 4). The results revealed that SiHa cells migrated significantly slower while transfected with TPX2-siRNA $(\mathrm{P}<0.05$; Fig. 4B). By contrast, there was no statistical difference between the untransfected $\mathrm{SiHa}$

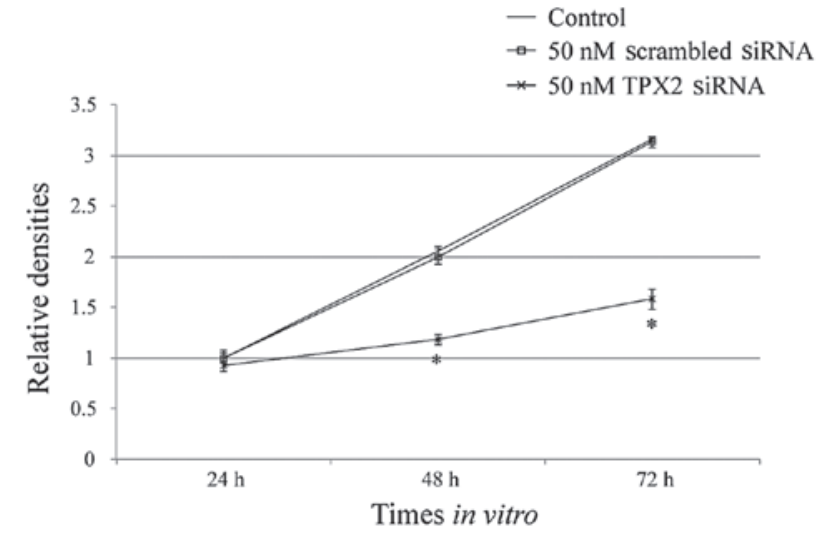

Figure 3. Cervical cancer proliferation was inhibited by TPX2 silencing. The proliferation capacity of SiHa cells lines was examined by an MTT assay. TPX2, targeting protein for Xenopus kinesin-like protein 2.

cells and the cells transfected with negative control siRNA $(\mathrm{P}>0.05)$.

TPX2 silencing reduces cervical tumor growth in vivo. Finally, we investigated whether the inhibitory effect of silencing TPX2 on cancer cell growth/migration in vitro would persist in vivo. Initially, SiHa cells were either untransfected (control), transfected with $50 \mathrm{nM}$ scrambled siRNA or transfected with $50 \mathrm{nM}$ TPX2 siRNA. Following this, the three groups of cells were injected into nude mice and the in vivo tumor growth one month after xenograft was examined. The results demonstrated that the TPX2 silencing significant reduced the growth of cervical tumor in nude mice (Fig. 5).

\section{Discussion}

TPX2 is a microtubule-associated protein that is important in the regulation of the cell cycle and mitosis, and functions in RanGTP-dependent manner $(9,10)$. Through cell mitosis, TPX2 interacts with downstream genes and proteins to locolize Aurora A to the microtubules of the mitotic spindle, and to induce Aurora A phosphorylation through an active structure $(1,11)$. In cancer biology, TPX2 was initially recognized as an oncogene factor amplified from chromosome 20q11.2 (12). Several studies have demonstrated that TPX2-induced tumor 
A
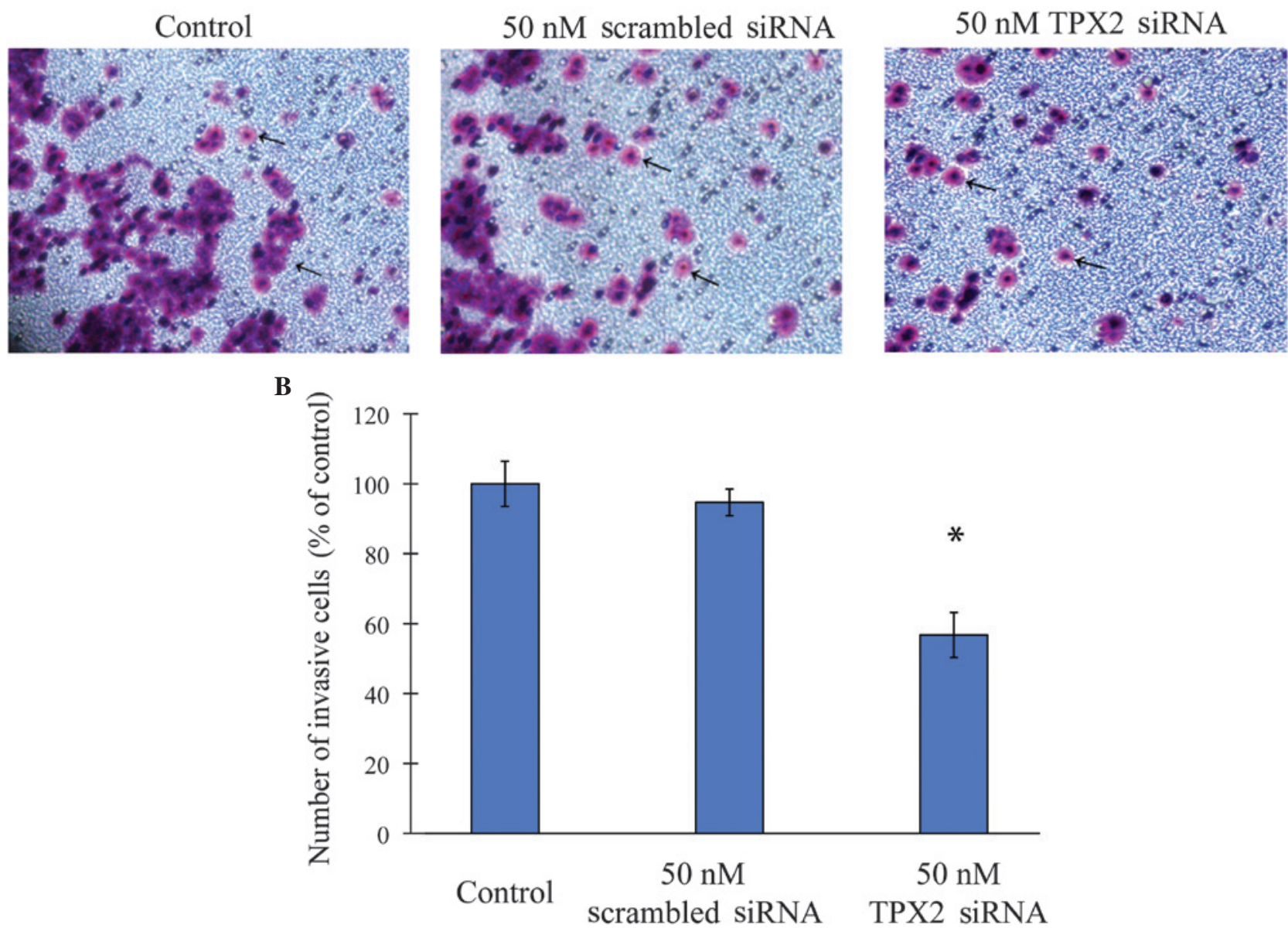

Figure 4. Cervical cancer cell migration was slowed by TPX2 silencing. The invasion of SiHa cells was examined by a Matrigel invasion assay. TPX2, targeting protein for Xenopus kinesin-like protein 2.
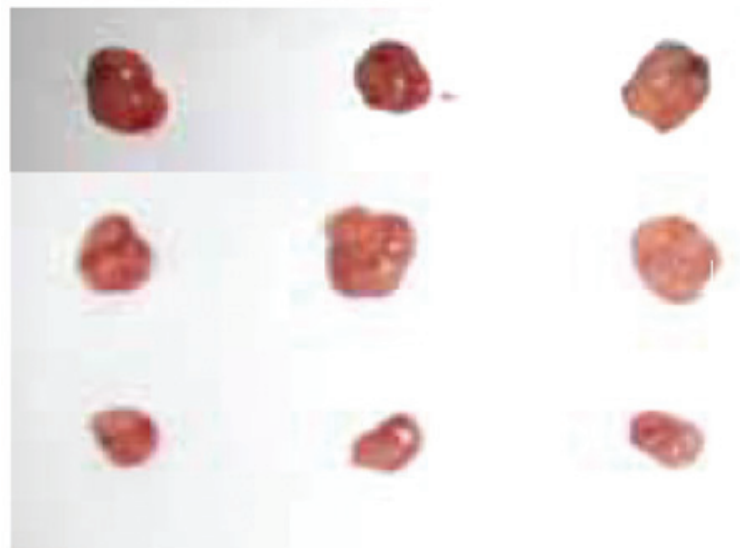

\section{Control}

\section{$50 \mathrm{nM}$}

scrambled siRNA

\section{$50 \mathrm{nM}$ TPX2 siRNA}

Figure 5. TPX2 regulated cervical tumor growth in vivo. Tumor sizes were measured 30 days following xenotransplantation. TPX2, targeting protein for Xenopus kinesin-like protein 2.

proliferation and inhibition of apoptosis, was upregulated in various tumorous tissue types, including lung, ovarian, pancreatic, breast and oral cancer (2,3,6,13-20).

In the present study, we demonstrated that TPX2 was expressed in cervical cancer carcinoma tissues in vivo and HeLa and SiHa cervical cancer cell lines in vitro, but not in the normal cervical epithelium tissues. TPX2 expression was then silenced in $\mathrm{SiHa}$ cells and it was revealed that this knockdown induced apoptosis, inhibited cell proliferation and slowed invasion. These results are consistent with previous studies that have demonstrated TPX2 actively regulated tumor growth in other cancer tissues $(21,22)$. Finally, in vivo tests were conducted and xenografted cervical tumors in nude mice were significantly reduced with TPX2 silencing.

To conclude, the results have suggested that TPX2 may become a new biomarker for cervical cancer diagnostics, and 
is a target that has potential for facilitating the development of more efficacious therapeutic methods to treat patients with cervical cancer.

\section{Acknowledgements}

The authors are grateful for the funding support from the Ministry of Health of China (no. W2011JZC32), the Natural Science Foundation of Jilin Province (no. 201215065) and the Development and Reform Commission of Jilin Province (no. 2011007-16)

\section{References}

1. Kufer TA, Silljé HH, Körner R, Gruss OJ, Meraldi P and Nigg EA Human TPX2 is required for targeting Aurora-A kinase to the spindle. J Cell Biol 158: 617-623, 2002.

2. Ma Y, Lin D, Sun W, et al: Expression of targeting protein for xklp2 associated with both malignant transformation of respiratory epithelium and progression of squamous cell lung cancer. Clin Cancer Res 12: 1121-1127, 2006.

3. Mohsenifar J, Almassi-Aghdam M, Mohammad-Taheri Z, et al: Prognostic values of proliferative markers ki-67 and repp86 in breast cancer. Arch Iran Med 10: 27-31, 2007.

4. Shigeishi H, Ohta K, Hiraoka M, et al: Expression of TPX2 in salivary gland carcinomas. Oncol Rep 21: 341-344, 2009.

5. Aguirre-Portolés C, Bird AW, Hyman A, Cañamero M, Pérez de Castro I and Malumbres M: Tpx 2 controls spindle integrity, genome stability, and tumor development. Cancer Res 72: 1518-1528, 2012.

6. Zhang L, Huang H, Deng L, et al: TPX2 in malignantly transformed human bronchial epithelial cells by anti-benzo[a] pyrene-7,8-diol-9,10-epoxide. Toxicology 252: 49-55, 2008.

7. Li L, Yang G, Ren C, et al: Glioma pathogenesis-related protein 1 induces prostate cancer cell death through Hsc70-mediated suppression of AURKA and TPX2. Mol Oncol 7: 484-496, 2013

8. Chang H, Wang J, Tian Y, Xu J, Gou X and Cheng J: The TPX2 gene is a promising diagnostic and therapeutic target for cervical cancer. Oncol Rep 27: 353-1359, 2012.

9. Gruss OJ and Vernos I: The mechanism of spindle assembly: functions of Ran and its target TPX2. J Cell Biol 166: 949-955, 2004.
10. Gruss OJ, Carazo-Salas RE, Schatz CA, et al: Ran induces spindle assembly by reversing the inhibitory effect of importin alpha on TPX2 activity. Cell 104: 83-93, 2001.

11. Bayliss R, Sardon T, Ebert J, Lindner D, Vernos I and Conti E: Determinants for Aurora-A activation and Aurora-B discrimination by TPX2. Cell Cycl 3: 404-407, 2004.

12. Tonon G, Wong KK, Maulik G, et al: High-resolution genomic profiles of human lung cancer. Proc Natl Acad Sci USA 102: 9625-9630, 2005.

13. Scharer CD, Laycock N, Osunkoya AO, et al: Aurora kinase inhibitors synergize with paclitaxel to induce apoptosis in ovarian cancer cells. J Transl Med 6: 79, 2008.

14. Warner SL, Stephens BJ, Nwokenkwo S, et al: Validation of TPX2 as a potential therapeutic target in pancreatic cancer cells. Clin Cancer Res 15: 6519-6528, 2009.

15. Stuart JE, Lusis EA, Scheck AC, et al: Identification of gene markers associated with aggressive meningioma by filtering across multiple sets of gene expression arrays. J Neuropathol Exp Neurol 70: 1-12, 2011.

16. Li B, Qi XQ, Chen X, et al: Expression of targeting protein for Xenopus kinesin-like protein 2 is associated with progression of human malignant astrocytoma. Brain Res 1352: 200-207, 2010.

17. Brizova H, Kalinova M, Krskova L, Mrhalova M and Kodet R: A novel quantitative PCR of proliferation markers (Ki-67, topoisomerase IIalpha, and TPX2): an immunohistochemical correlation, testing, and optimizing for mantle cell lymphoma. Virchows Arch 456: 671-679, 2010.

18. Satow R, Shitashige M, Kanai Y, et al: Combined functional genome survey of therapeutic targets for hepatocellular carcinoma. Clin Cancer Res 16: 2518-2528, 2010.

19. Shigeishi H, Fujimoto S, Hiraoka M, et al: Overexpression of the receptor for hyaluronan-mediated motility, correlates with expression of microtubule-associated protein in human oral squamous cell carcinomas. Int J Oncol 34: 1565-1571, 2009.

20. Smith LT, Mayerson J, Nowak NJ, et al: 20q11.1 amplification in giant-cell tumor of bone: Array CGH, FISH, and association with outcome. Genes Chromosomes Cancer 45: 957-966, 2006.

21. Wei P, Zhang N, Xu Y, et al: TPX2 is a novel prognostic marker for the growth and metastasis of colon cancer. J Transl Med 11: 313, 2013.

22. Morgan-Lappe SE, Tucker LA, Huang X, et al: Identification of Ras-related nuclear protein, targeting protein for xenopus kinesin-like protein 2 , and stearoyl-CoA desaturase 1 as promising cancer targets from an RNAi-based screen. Cancer Res 67: 4390-4398, 2007. 